\title{
Transient Thermographic Heat Path Analysis using Spatially Resolved Thermal Equivalent Networks
}

\author{
by Nils J. Ziegeler*, Peter W. Nolte**, and Stefan Schweizer ${ }^{*, * *}$
}

\begin{abstract}
* Faculty of Electrical Engineering, South Westphalia University of Applied Sciences, Lübecker Ring 2, 59494 Soest, Germany, ziegeler.nilsjonas@fh-swf.de, schweizer.stefan@fh-swf.de

${ }^{* *}$ Fraunhofer Application Center for Inorganic Phosphors, Branch Lab of Fraunhofer Institute for Microstructure of Materials and Systems IMWS, Lübecker Ring 2, 59494 Soest, Germany, peter.nolte@imws.fraunhofer.de
\end{abstract}

\begin{abstract}
In this work, an evaluation method for transient thermal measurements is presented. It is based on a onedimensional heat path analysis technique common for the thermal characterization of electronic components. This technique is generalised to obtain spatially resolved values for thermal resistances as well as thermal capacities via infrared thermography. The analysis of recorded image sequences yields the "time constant spectrum", which enables for a detailed insight into the physical parameters describing the heat flow dynamic of the device. As a proof of concept, a $4 \times 4$ LED array is investigated and the experimental results are analysed.
\end{abstract}

\section{Introduction}

Electronic components are thoroughly analysed in every stage of their design by ever more complex finite element and fluid dynamic simulations. Accordingly, the demand for model verification increases. For thermal design, the physical parameters of prototypes are determined to quantify the heat flow behaviour within the component. Nevertheless, thorough quantitative testing of these parameters during production is also desirable.

For isolated electronic components, such as light-emitting diodes (LEDs), the "Network Identification by Deconvolution" method is well established to characterise a one-dimensional heat flow path [1]. A thermally sensitive electric property, such as the forward voltage of a p-n-junction, is used for a time-resolved measurement of the component's response to a jump of the dissipated heat power. By evaluation of the corresponding time series, the thermal resistances as well as the thermal capacities of the heat path can be determined. The idea of this work is to transfer this technique to infrared thermography. Infrared images provide spatially resolved temperature data of the entire surface of a device under test. In this way, more detailed information about the device can be captured. The presented method is similar to "Thermal Signal Reconstruction" with respect to the evaluation of logarithmic thermographic image sequences [2]. Its merits are the use of a non-parametric model and physical parameters that can form a basis for further analysis.

\section{Theoretical Background}

For transient thermal measurements, the device is brought to an elevated temperature first. This can be achieved either by self-heating during operation of the device or by applying external heat to the surface until a thermal equilibrium is reached. Then, the heating power is instantly turned off and the thermal response during relaxation back to ambient temperature is recorded.

The thermal impedance, $Z_{\mathrm{th}}(t)$, captures the step response of the device under test. It contains all information about the thermal resistances, $R_{\mathrm{th}}$, as well as the thermal capacities, $C_{\mathrm{th}}$, along the heat path. It can be used to calculate a well-defined thermal equivalent network, which describes the heat path from the LED to the heat sink. Here, the heat is assumed to be conducted via a one-dimensional path down to the heat sink. The validity of this approximation depends on the system in question. Heat losses via parasitic heat paths, such as convection, can also be accounted for [3]. An equivalent network is an electrical circuit that mimics the heat conduction behaviour within the device. Thermal resistances as well as thermal capacities are attributed to their electrical equivalents; temperature differences correspond to voltages.

In the following, the method of "Network Identification by Deconvolution", which allows calculating such an equivalent network from a given temperature curve, is described $[4,5]$. On one end of the path is a heat source that raises the device temperature, $T$, to an elevated initial value, $T_{0}$. The heating power, $P$, describes the magnitude of the subsequent power step. The corresponding thermal impedance, $Z_{\mathrm{th}}(t)$, is defined as the temperature difference relative to the initial temperature divided by the heating power, $P$. The thermal impedance is usually expressed in logarithmic time, $z=\ln (t)$. The resulting function is called $a(z)$, i.e.,

$$
Z_{\mathrm{th}}(t)=\frac{T_{0}-T(t)}{P} \quad \text { and } \quad a(z)=Z_{\mathrm{th}}(t=\exp (z))
$$


A thermal equivalent circuit suitable to describe the thermal behaviour is an $\mathrm{RC}$ one-port. The Foster network displayed in Fig. 1 represents such a network. It has three RC components which correspond to three different material layers. Each layer is represented by a thermal resistance and a thermal capacity connected in parallel.

The behaviour of a network is characterized by its electrical impedance which is usually written as a complex number. The real part corresponds to the ohmic resistance while the imaginary part represents the reactance of the network. The current response of a circuit to a sinusoidal voltage is completely defined by the complex impedance. However, for the electrical analogy circuit investigated here, a sinusoidal voltage, i.e. a sinusoidal temperature difference, is not applicable, since a step response is measured. To account for a non-sinusoidal input, the impedance is generalized by introducing the complex frequency, $s$, instead of $\mathbf{i} \omega$. This complex frequency is related to time-domain signals via Laplace transformation.

The generalised $s$-plane impedance, $Z(s)$, of the network contains all information about the response of the electrical network to arbitrary input. It is calculated by combining the impedances of the subcomponents which are connected either in parallel or in series. The final goal is to relate the electrical impedance, $Z(s)$, to the thermal impedance, $Z_{\mathrm{th}}(t)$. It has to be noted that the resistances as well as the capacities in a Foster network do not correspond to physically measurable values. To obtain these, the network is transformed to a Cauer network (Fig. 2) with the same $Z(s)$, as it will be discussed below.

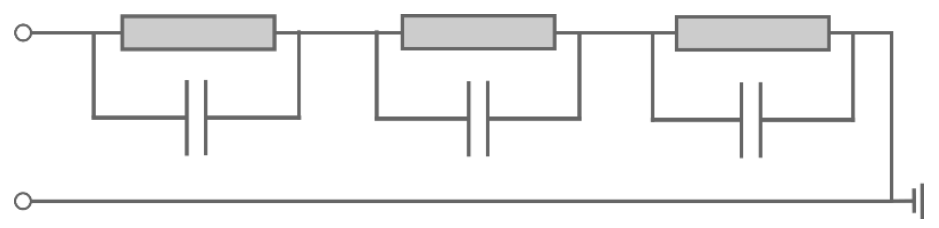

Fig. 1. Electrical equivalent Foster network with three different layers. Each layer comprises a thermal resistance and a thermal capacity connected in parallel.

The Foster network has the useful quality that it can be directly derived from the logarithmic time constant spectrum, $R(\zeta)$. The latter can be derived from the network impedance, $Z(s)$, by taking its imaginary part along the negative real axis, as it can be seen in Eq. (2).

$$
R(\zeta)=\frac{1}{\pi} \operatorname{lm}(Z(s=-\exp (-\zeta))) \quad \text { with } \quad \zeta=\ln (\tau)
$$

Discretizing the time constant spectrum, which is mandatory in computer evaluations anyway, yields the layers of the equivalent network. The values of the electrical capacities, $C$, in the Foster network are derived via the definition of the time constant, $\tau=R \cdot C$.

The time constant spectrum is connected to the derivative of the thermal impedance $a(z)$ with respect to $z$ by convolution with a weight function, $w_{z}(z)$, as shown in Eq. (3). To obtain the time constant spectrum, a deconvolution has to be performed.

$$
\begin{aligned}
\frac{\mathrm{d}}{\mathrm{d} z} a(z) & =\left(R \otimes w_{z}\right)(z) \\
& =\int_{-\infty}^{+\infty} R(\zeta) \exp (z-\zeta-\exp (z-\zeta)) \mathrm{d} \zeta
\end{aligned}
$$

The weight function $w_{z}(z)$ is defined as

$$
w_{z}(z)=\exp (z-\exp (z))
$$

To obtain physical parameters relating to the small elements of the quantised heat path, the Foster network is transformed into an equivalent Cauer network (Fig. 2). 


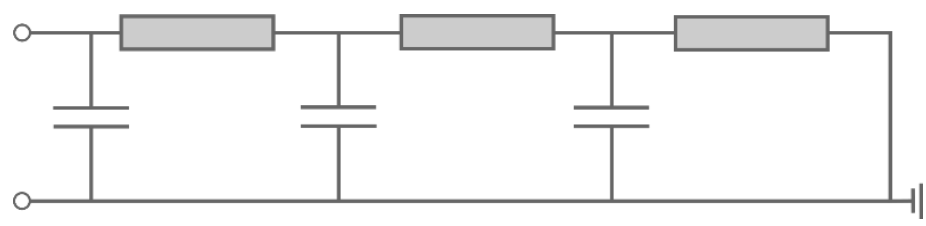

Fig. 2. Electrical equivalent Cauer network with three different layers. In each layer, the capacity is connected to the ground. All resistances are connected in series. The values for the resistances as well as the capacities in the circuit can be directly identified with their thermal counterparts in the device under test.

The transformation can be achieved by continued polynomial division, as described in $[5,6]$. The idea is to separate parallel capacities and serial resistances from the $n$-layer network. To this end, Eq. (5) is applied recursively until the entire Cauer network has been constructed. In each iteration, the order of the remaining network, $Z_{n-1}(s)$, is reduced by one. The starting point is the impedance, $Z(s)$, of the Foster network.

$$
\frac{1}{Z_{n}(s)}=s C_{n}+\frac{1}{R_{n}+Z_{n-1}(s)}
$$

The thermal resistances, $R_{n}$, as well as the thermal capacities, $C_{n}$, both derived via Eq. (5), correspond to the physically measurable values of the various elements comprising the heat path. The thermal structure function is defined as the thermal capacity integrated along the heat path as a function of the thermal resistance integrated along the heat path starting at the power source. Structure functions diverge as the integrated thermal resistance reaches the steady-state thermal resistance, $R_{\text {th }}$, as the surrounding bath has an approximately infinite thermal capacity.

\section{Experimental Details}

The thermographic setup consists of an InfraTec ImageIR 8380S (spectral range 2.0-5.7 $\mu \mathrm{m}$ ). It has a cooled indium antimonide focal plane array snapshot detector with a noise equivalent temperature difference of less than $25 \mathrm{mK}$ at detector temperatures of approximately $70 \mathrm{~K}$. The geometric resolution is $640 \times 512 \mathrm{px}$, the sampling rate is $200 \mathrm{~Hz}$. The camera and the LED array are connected to a programmable controller for frame synchronization.

The sample investigated is a commercially available LED array comprised of 16 LEDs in a $4 \times 4$ arrangement. The equilibrium temperature distribution (without emissivity correction) is shown in Fig. 3. Electrically, the LED array consists of four strings, which are connected in parallel. Each string comprises four LEDs and one resistor at the bottom. The array is operated at $0.5 \mathrm{~A}$ and $5 \mathrm{~V}$.

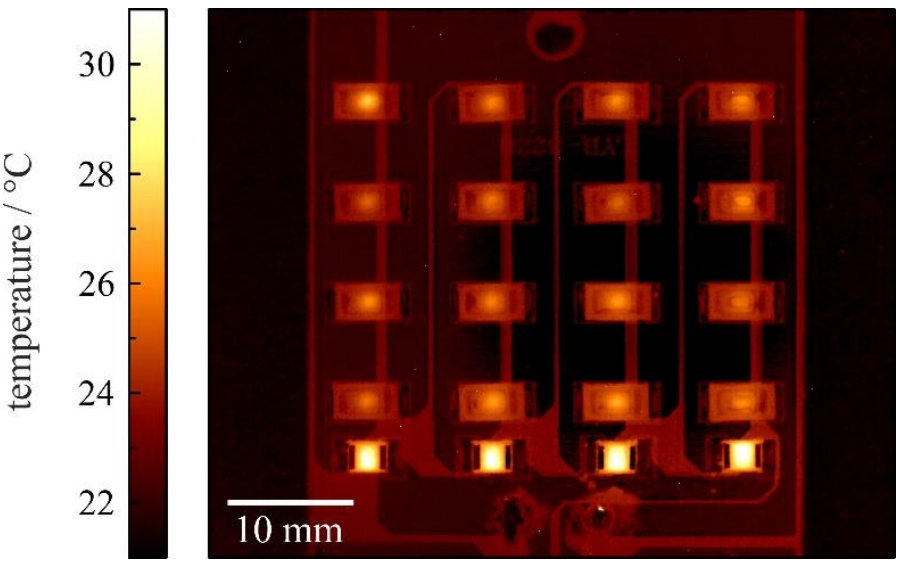

Fig. 3. The device under test is a $4 \times 4$ LED array. At the bottom, a row of four resistors is visible.

For the experiment, the LED array is mounted on a temperature-controlled heat sink. Energy dissipation mainly occurs in the semiconductor, in the phosphor, and in the resistors. Commonly, the junction temperature is monitored via the forward voltage to determine the LED's thermal structure function, i.e., only a single temperature value is provided for each LED. In this work, the thermal relaxation behaviour is analysed pixelwise for the entire LED array, and a spatially resolved map of time constants is obtained. The fact, that temperature measurements by infrared thermography do not depend on the design of the electrical circuit, represents an additional benefit of this technique.

In Fig. 3, the equilibrium temperature distribution already hints that the performance of the various LEDs differs. An image of the static temperature distribution, however, might be misinterpreted with respect to the quality of the thermal 
contact or the heat path itself, since heat spreading effects of neighbouring components cannot be distinguished unambiguously. Here, dynamic measurements are advantageous, since they can resolve heat spreading effects from individual LED effects. For the experiment, the LED array is self-heated to an elevated equilibrium temperature. This takes in the order of $100 \mathrm{~s}$. Then, the array is switched off.

\section{Results}

For the results presented here, the method of "Network Identification by Deconvolution" is applied to the temperature curve of each pixel of the infrared image separately. For all calculations, both power step and emissivity are assumed to be unity.

To monitor the thermal relaxation with maximum temporal resolution, the highest sampling frequency is used at the beginning of the experiment. This is particularly important in the case of fast relaxation processes. During the measurement, the sampling frequency is lowered for the sake of reduced data volume. Reducing the sampling frequency with increasing measurement time even further does not affect the measurement accuracy significantly, since the data analysis is performed on a logarithmic time scale. The measurement points lie increasingly close to each other, as it can be seen in Fig. 4. As a consequence, the time-series data in logarithmic time is sparse at the beginning. This makes the calculation of derivatives challenging, in particular in the case of noisy data.

In the following, the evaluation is exemplified by temperature data from an arbitrary pixel. The corresponding thermal impedance, as defined in Eq. (1), is shown in Fig. 4. As the power step is assumed to be unity, the thermal impedance directly corresponds to the temperature difference. Because of the low absolute temperature differences, the signal-to-noise ratio of the detector is relatively low. The discrete steps in the impedance values are due to the quantization effects of the infrared detector.

A locally weighted scatterplot smoothing (LOWESS) filter, also known as Savitzky-Golay filter, shows the underlying trend. The thermal relaxation, as shown in Fig. 4, basically happens in two different time regimes: The first regime lies in the range of a few seconds, while the second one is in the range of a few tens of seconds. Towards the end of the measurement, the thermal impedance slightly decreases, i.e. the temperature increases. This is presumably due to a drift in the ambient temperature. The observed temperature rise amounts to approximately $40 \mathrm{mK}$ over a period of more than $300 \mathrm{~s}$.

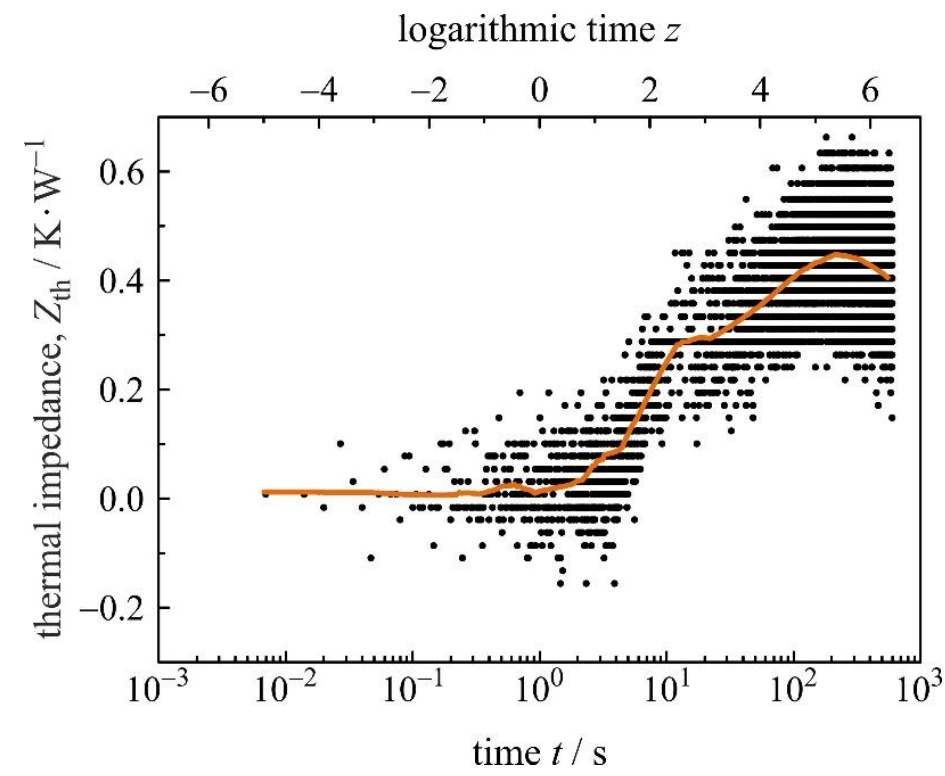

Fig. 4. Thermal impedance, $Z_{\text {th }}$ of an arbitrary pixel. The points correspond to the noisy data as observed by the infrared detector. The orange line represents a LOWESS curve.

In the next step, the derivative of the thermal impedance is calculated for the deconvolution, as described in Eq. (3). Since the LOWESS curve is based on polynomials to capture local trends, it is possible to obtain the corresponding derivatives by directly taking the derivative of the polynomials. The calculated derivative spectrum is shown in Fig. 5 . 
logarithmic time $z$

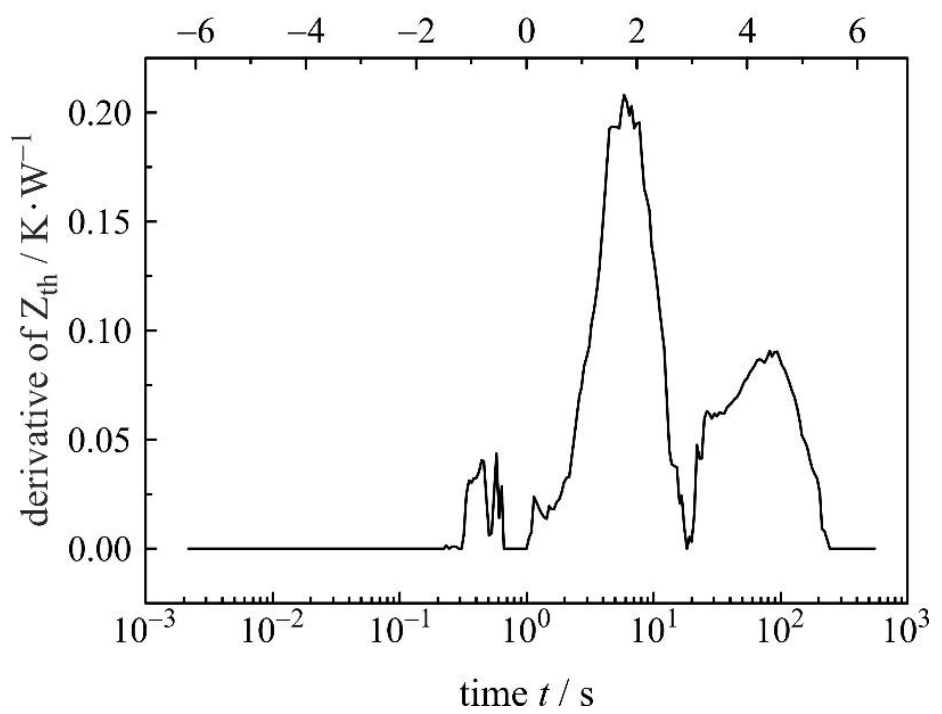

Fig. 5. Derivative of the thermal impedance, $Z_{\text {th }}$ as shown in Fig. 4. The derivative of the LOWESS curve is taken at points which are equally spaced on a logarithmic time scale.

As negative derivative values are unphysical (this would correspond to self-heating without power applied) they are set to zero. The further evaluation requires equally spaced data which are not provided by the measurement system. Thus, the regression is evaluated on a new grid with 275 points that are evenly distributed in logarithmic time.

The deconvolution is performed via Bayesian deconvolution [7]. This has the advantage (as opposed to Fourier space deconvolution) to conserve the area under the curve and to produce non-negative results. To calculate the time constant spectrum from the derivative data, the Bayesian iteration is performed with 1000 steps. The result is shown in Fig. 6. The spectrum corresponds to a thermal equivalent network with 275 layers, most of which have zero or close to zero thermal resistance.

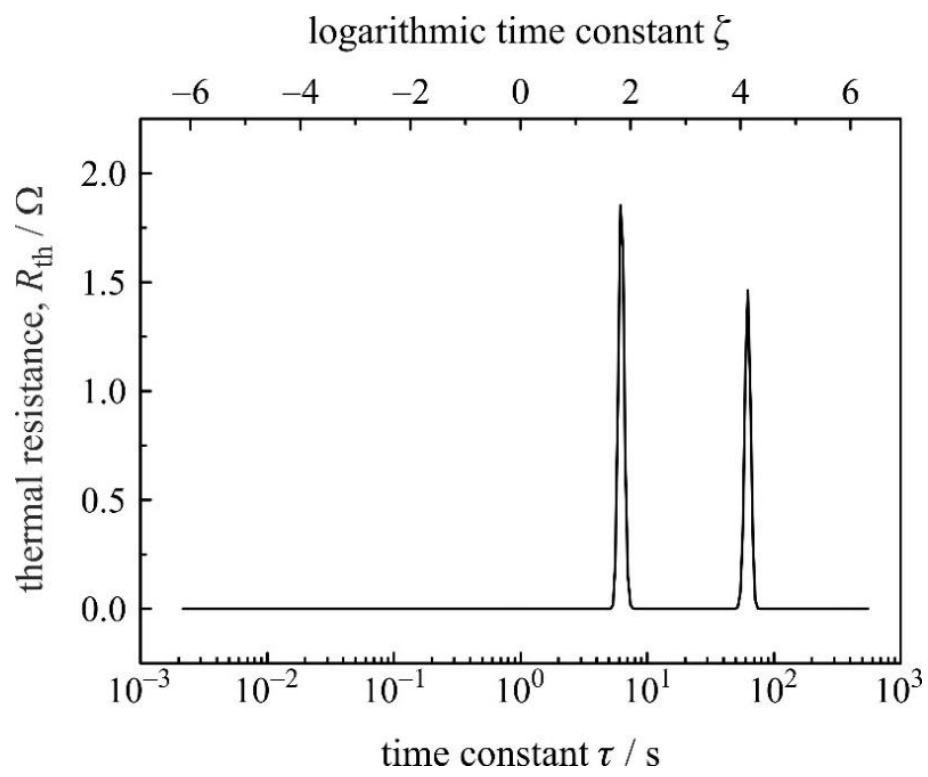

Fig. 6. Time constant spectrum calculated via Bayesian deconvolution from the derivative data, as shown in Fig. 5.

As described above for an arbitrary pixel, identical calculations are performed for the temperature data of each pixel of the entire image sequence. Fig. 7 shows snapshots of the spatially resolved time constant spectrum for three different time constants. 

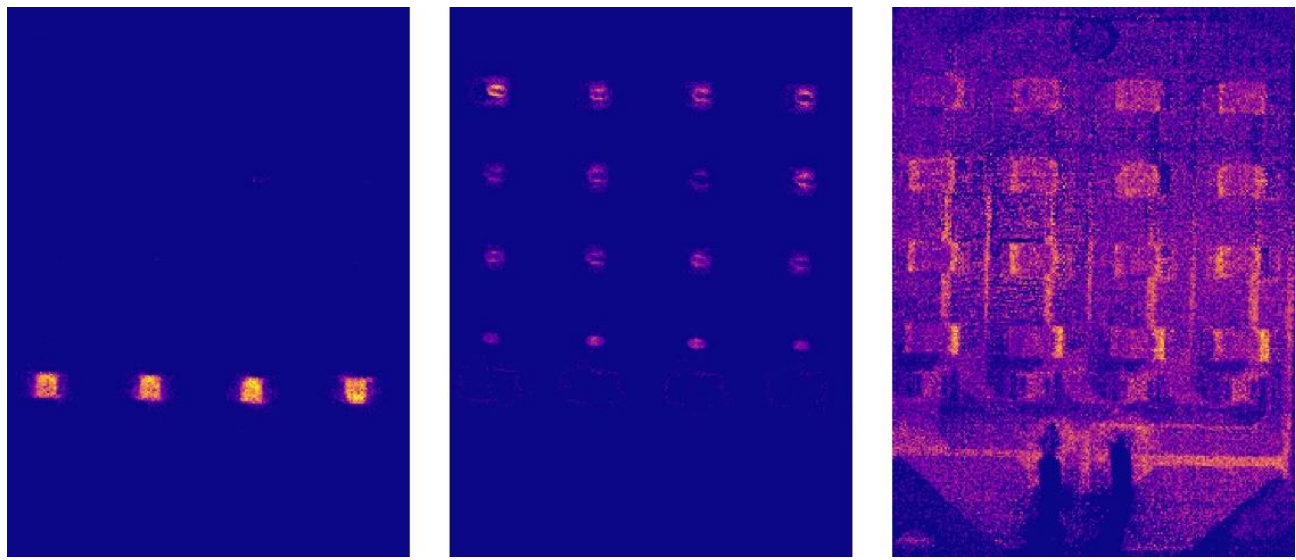

Fig. 7. Spatially resolved amplitude of the time constant spectrum for different logarithmic time constants: $\tau=256 \mathrm{~ms}, \tau=554 \mathrm{~ms}$ and $\tau=5.62 \mathrm{~s}$ (from left to right). The images comprise the thermal relaxation behaviour of all components and layers of the LED array.

The left image of Fig. 7 shows the LED array for a short time constant of $\tau=256 \mathrm{~ms}$. Here, the four resistors are the only components showing a significant temperature change, revealing that they are the fastest reacting components. The image in the middle represents an intermediate time constant of $\tau=554 \mathrm{~ms}$. The resistors are barely noticeable, the thermal relaxation of the LEDs is inhomogeneous. Each LED shows a ring-shaped structure, which is possibly due to the fact that the centre of the LED relaxes much faster than the edges. For the long time constant of $\tau=5.62 \mathrm{~s}$, as shown on the right, the thermal relaxation is more homogeneous because the heat flow from the circuit board to the heat sink is the dominant contribution.

Notably, the top left LED shows a higher average intensity than the other LEDs at an intermediate time constant of $\tau=554 \mathrm{~ms}$, while for shorter and longer time constants the behaviour of the LED does not stick out. This indicates a faulty heat path for the corresponding LED. The magnitude of this defect is only vague in the steady-state temperature image, as shown in Fig. 3, because the ambient heating of the resistors at the bottom of the array induce an overall temperature gradient on the PCB. Analysing the transient behaviour allows separating these effects.

\section{Conclusion}

A method to determine the spatially resolved time constant spectrum of electronic devices is presented exemplarily for an LED array. To analyse the thermal step response of the device under test, the object is first heated to an elevated equilibrium temperature. Subsequently, the thermal relaxation is monitored by infrared thermography. From the recorded image series, time constant spectra are calculated. The multitude of time constant spectra is compiled into a two-dimensional image sequence for the object. These images reveal details of the heat path as well as the influence of the various components on the thermal relaxation behaviour. The method works contactless and enables a heat path analysis at an early stage of a development and production process when no electric contact is available yet.

\section{REFERENCES}

[1] JEDEC JESD 51-51, "Implementation of the Electrical Test Method for the Measurement of Real Thermal Resistance and Impedance of Light-Emitting Diodes with Exposed Cooling", JEDEC, 2012

[2] S. M, Shepard, et al., "Reconstruction and enhancement of active thermographic image sequences", Optical Engineering, 2003. 42(5): p. 1337-1342

[3] M. Rencz, A. Poppe, E. Kollar, S. Ress, V. Szekely, "Increasing the accuracy of structure function based thermal material parameter measurements", in IEEE Transactions on Components and Packaging Technologies, 2005, 28:1, 51-57, DOI: 10.1109/TCAPT.2004.843204

[4] V. Szekely, "Identification of RC networks by deconvolution: chances and limits", in IEEE Transactions on Circuits and Systems I: Fundamental Theory and Applications, 1998, 45:3, 244-258. DOI: 10.1109/81.662698

[5] JEDEC JESD 51-14, "Transient Dual Interface Test Method for the Measurement of the Thermal Resistance Junction to Case of Semiconductor Devices with Heat Flow Through a Single Path", JEDEC, 2010

[6] Y. C. Gerstenmaier, W. Kiffe and G. Wachutka, "Combination of thermal subsystems modeled by rapid circuit transformation," 2007 13th International Workshop on Thermal Investigation of ICs and Systems (THERMINIC), 2007, 115-120, DOI: 10.1109/THERMINIC.2007.4451758

[7] T. J. Kennett, W. V. Prestwich, A. Robertson, "Bayesian deconvolution I: Convergent properties", Nuclear Instruments and Methods, 1978, 151:1-2, 285-292, DOI:10.1016/0029-554X(78)90502-5 